\title{
A narrative review of the interpretation of guidelines for the treatment of infective endocarditis
}

\author{
Umberto Benedetto ${ }^{1}$, Sanjeet Singh Avtaar Singh ${ }^{2,3}$, Cristiano Spadaccio ${ }^{2,3}$, Marc R. Moon ${ }^{4}$, \\ Francesco Nappi ${ }^{5}$
}

${ }^{1}$ Department of Cardiothoracic Surgery, Bristol Heart Institute, University of Bristol, Bristol, UK; ${ }^{2}$ Department of Cardiac Surgery, Golden Jubilee National Hospital, Glasgow, UK; ${ }^{3}$ Institute of Cardiovascular and Medical Sciences, University of Glasgow, UK; ${ }^{4}$ Department of Cardiac Thoracic Surgery, Washington University School of Medicine, Saint Louis, Missouri, USA; ${ }^{5}$ Department of Cardiac Surgery, Centre Cardiologique du Nord de Saint-Denis, Paris, France

Contributions: (I) Conception and design: U Benedetto, F Nappi; (II) Administrative support: None; (III) Provision of study materials or patients: None; (IV) Collection and assembly of data: F Nappi; (V) Data analysis and interpretation: F Nappi; (VI) Manuscript writing: All authors; (VII) Final approval of manuscript: All authors.

Correspondence to: Francesco Nappi, MD. Department of Cardiac Surgery, Centre Cardiologique du Nord, 36 Rue des Moulins Gémeaux, 93200 Saint-Denis, Paris, France. Email: francesconappi2@gmail.com.

\begin{abstract}
The recommendations of the current guidelines and the position papers of professional societies from the European Society of Cardiology/European Society of Cardiothoracic Surgeons (ESC), the American College of Cardiology/American Heart Association/Society of Thoracic Surgeon (ACC/ AHA/STS) and American Association of Thoracic Surgeon (AATS) regarding management of patients with valvular heart endocarditis were updated over the past decade. However, some of the recommendations appear to contradict one another. Given the changing paradigms on how the disease manifests, our aim was to review the respective guidelines and highlight these differences whilst drawing attention to the subsequent studies from which they were derived. In particular, concerns regarding antibiotic prophylaxis and therapy, imaging modality for diagnosis and follow-up, cerebrovascular sequalae and timing of surgery are appraised in detail. We also identified the novel techniques used such as transcatheter therapies and advances in imaging modalities used for diagnosis and treatment of this condition. The lack of randomised control trials (RCTs) does raise several issues regarding applicability of findings in day-to-day practice. Therefore, the focus of upcoming studies should be on clearly defined multicenter RCTs to provide more robust evidence for the management and treatment of infective endocarditis as future guidelines will be based on the outcomes of these trials.
\end{abstract}

Keywords: Infective endocarditis; guidelines; treatment and management

Submitted May 05, 2020. Accepted for publication Jul 10, 2020.

doi: 10.21037/atm-20-3739

View this article at: http://dx.doi.org/10.21037/atm-20-3739

\section{Introduction}

The recommendations of the current guidelines and the position papers of professional societies from the European Society of Cardiology/European Society of Cardiothoracic Surgeons (ESC), the American College of Cardiology/ American Heart Association/Society of Thoracic Surgeon (ACC/AHA/STS) and American Association of Thoracic
Surgeon (AATS) regarding management of patients with valvular heart endocarditis were updated in 2014 $(1,2), 2015$ (3) and again in $2016(4)$ and $2017(5,6)$ respectively. There are important differences within the recommendations relating to the management of patients with heart valve endocarditis (HVE), as with updated evidence via the publication of several reports from professional society recommendations. This study aims to 


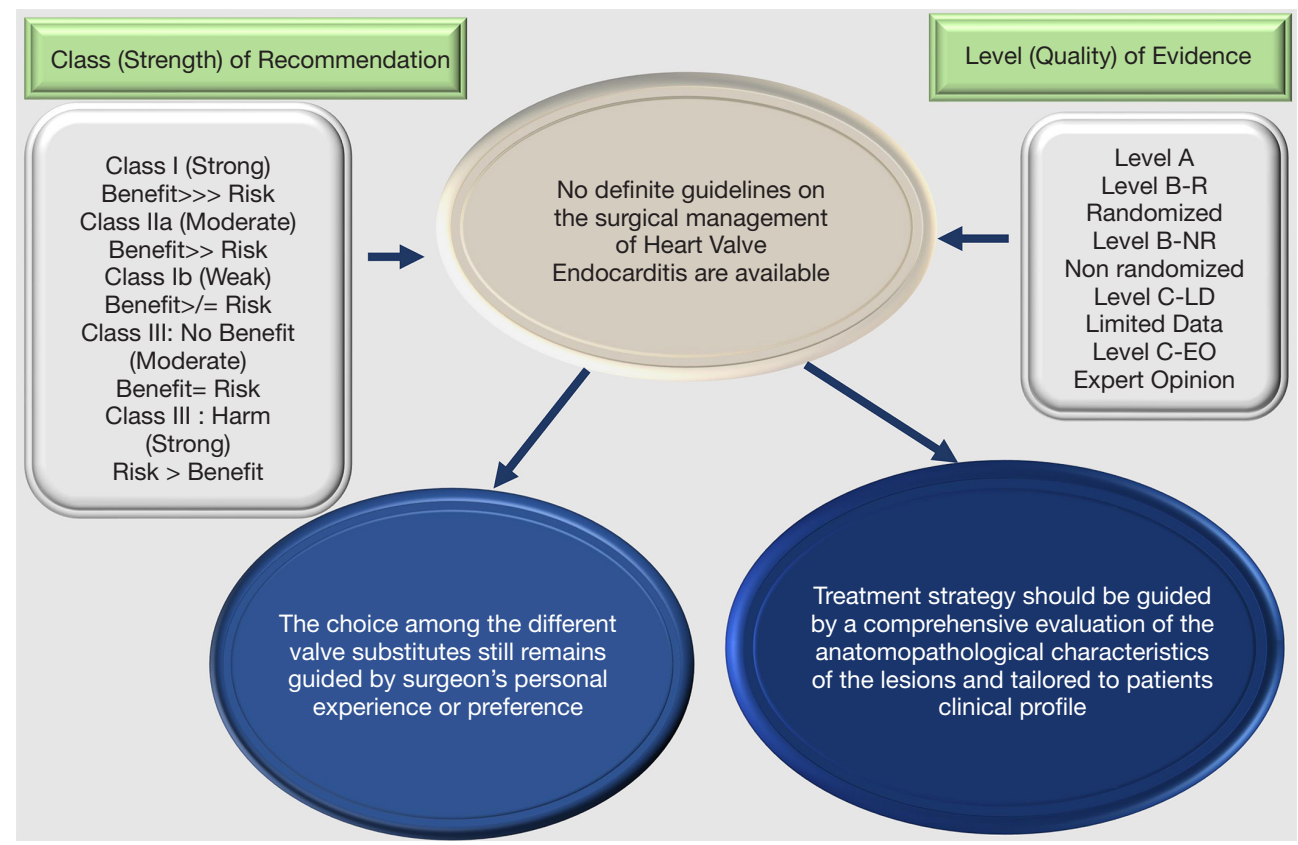

Figure 1 Class of recommendation (COR) and the level of evidence (LOE) that establishes the degree of choice for a treatment.

compare the recommendations by current guidelines and the position papers of professional societies with regards to management of patients with HVE. This review is focused on the differences between the two guidelines developed, by cardiologists and cardiac surgeons, and its purpose is to summarize new data that addresses some of the ongoing debates. The authors present the following article in accordance with the Narrative Review reporting checklist (available at http://dx.doi.org/10.21037/atm-20$3739)$.

\section{Class of recommendation (COR) and level or quality of evidence}

The implementation of the COR and level of evidence (LOE) for the clinical strategy, intervention, treatment or diagnostic tests in patient care with HVE is generally addressed in prophylaxis, diagnosis and management which includes the choice of the best valve substitute and the timing for surgery as well as the prevention of complications.

The focus of the guidelines for the management of patients with infectious disease of the heart valves is represented by the diagnosis and management of adult patients in whom the infection of the heart valve has occurred. The field of HVE is rapidly progressing, with updated knowledge about the natural history of patients with valvular endocarditis, diagnostic imaging advances and improvements in catheter-based interventions alongside standard surgery. Several randomized controlled trials (RCTs) were highlighted in the guidelines, specifically with regards to the results on the timeliness of intervention, on the choice of antibiotic treatment and prevention of neurological complications. The main areas of change include indications for transcatheter aortic valve replacement (TAVR), for sutureless and for better management of patients with prosthetic valve endocarditis.

The evidence also highlights a significant variability in HVE presentation and difficulty in establishing unequivocal clinical practice. Indeed, the recommendations from the position papers of professional societies are based on observational cohort studies or few individual RCTs rather than multicenter RCTs or patient-level combined analysis of RCTs. Figure 1 denotes the COR and the LOE that establishes the degree of choice for a treatment. It is important to point out that COR and LOE are dictated separately. When a recommendation is graded as Level C it is not universally considered as a weak recommendation. In fact, many of the therapeutic addresses that are given in the guidelines and professional societies recommendations originate from non-randomized studies. However, RCTs provide clear consensus for the application of a treatment 
and therefore determine its effectiveness and safety (Figure 1).

The literature is scarce on data from randomized studies classified as LOE A that address a systematic approach not only for the choice of ideal substitute for IE but also with regards to the choice of prosthetic heart valve. It is indisputable that the LOE is useful to orient the timing and choices of the prosthetic heart valve in endocarditis. Analyzing the guidelines, we can observe that $6.7 \%$ of evidence by ACC/AHA $(1,6)$ is Class I and $3 \%$ of ESC $(3,5)$ is Class IIa. The LOE B based on recommendations is measured at $57 \%$ for ACC/AHA and $18 \%$ for ESC while the $\mathrm{LOE} \mathrm{C}$ based on recommendations is measured at $36.3 \%$ for ACC/AHA and to $79 \%$ for ESC guidelines

\section{Controversies regarding management of patients with HVE}

\section{Effects of changing guidelines on the incidence of IE}

In the case of IE, the AHA/ACC guidelines for antibiotic prophylaxis are restrictive in the United States. Prophylaxis is indicated in patients who have received a prosthetic valve, CHD with an episode of IE, or in recipients of cardiac transplantation in whom valvulopathy has developed (7). DeSimone et al. $(8,9)$, using data from the Rochester Epidemiological Project, analyzed the incidence of IE due to viridans group streptococci before and after the change of prophylaxis guidelines. The authors found no significant increase in the incidence and, in contrast, emphasized a decrease of 3.6 per 100,000/year from 1999 to 2002 to 1.5 per 100,000 person-years from 2011-2013. Similarly, two reports performed on the Canadian population (10) and the United States (11) who underwent prophylactic treatment for IE, found no evidence for a change point in the incidence of IE to coincide with modified ACC/AHA guidelines. The most relevant studies that contrast with AHA/ACC are two national epidemiological studies from the United States and the United Kingdom which raised some concerns.

Pant et al. (12), using the Nationwide Inpatient Sample, revealed that although they showed a statistically significant increase in the incidence of IE caused by streptococci, they were unable to identify significant changes in the trend, with a growing curve, both in total admissions and in the number of patients with IE due to $S$ aureus. It should be noted that this study included non-homogeneous groups because of the incidence calculations in which both groups, non-viridans streptococci and enterococci were evaluated. The weakness lies in the fact that the new change point analysis was not carried out to confirm that the change in the rate coincided with the ACC/AHA amended guidelines. In addition, the investigators did not have access to the prescription data of antibiotic prophylaxis to confirm that this rate had decreased.

In the UK (13) there is a marked restriction on the use of antibiotic prophylaxis. The national stance, since March 2008 , is to advise against the use of antibiotic prophylaxis. Initial analysis did not report an increase in the incidence of IE. The effects of the restriction on the use of antibiotics in IE prophylaxis are published in a 2015 study (14). In an extended analysis, the hospital discharge diagnoses were examined and reported by the National Health Service until 2013. Antibiotic prophylaxis decreased from 10,900 prescriptions per month to 2,236 prescriptions per month after the introduction of the UK National Institute for Health and Care Excellence (NICE) guidelines. In parallel, there was a significant increase (above the expected trend) in the number of cases of IE, of 0.11 cases per 10 million people (or another 35 cases in England) per month. The statistical analysis identified June 2008 (3 months after the implementation of the new guidelines for the use of antibiotic prophylaxis) as a point of change, but it was not possible to confirm that these cases were due to oral streptococci because the microbiological data was not available.

A large restriction on the use of antibiotic prophylaxis for IE emerges from data from studies in France, where antibiotic prophylaxis was limited to high risk groups as early as 2002. The analysis used a very precise investigative approach to collect data on all cases of IE in different regions $(15,16)$. The two studies showed that the incidence of IE in three years of investigation (1991, 1999 and 2008) was stable at 35, 33 and 32 cases per million, suggesting that there was no significant change since the limitation of oral antibiotic prophylaxis. Importantly, the number of cases caused by oral streptococci was also stable. These data are mainly established on the basis of large observational studies and have not reported a causal link between restriction of antibiotic prophylaxis and incidence of IE. One of the reasons that causes a confusion is that the inferior clinical results associated with reduced use of antibiotic for prophylaxis of IE that have been reported in the registries have not been replicated in RCTs. There is growing concern that observational studies can be biased in favor of an increase in the number of IE after implants of the device, 
although this factor has been adapted in some studies, by unmatched confounders related to the unmeasurable (and un-matchable) judgment of the operating cardiologist. Despite long-term controversy and difficulties with observational data, randomized trials are highly unlikely due to cost, logistics, and ethical debate about real equipoise exists to allow the conduction of a placebo-controlled study.

Currently the ACC/AHA (1) and the European Society of Cardiology (ESC) $(3,5,17)$ have a pragmatic approach in which the indications are to limit prophylaxis to individuals at higher risk based on their underlying heart conditions. We believe that this approach represents a correct balance between the risks and benefits for the individual and the population of using antibiotics. It is important to note that the indications do not include patients who present with non-cardiac risk factors (e.g., those who are immunodeficient) and who may be at increased risk of IE and poor outcome if the disease develops. There is an emergence of data that suggests in these patients, a tailored approach to individual patients remains appropriate, according to clinical circumstance $(18,19)$.

\section{Routine imaging}

It is agreed that early pre-operative imaging is recommended in all patients to establish a baseline for the diagnosis and treatment of infective endocarditis. There is a general consensus to the use of echocardiography, that remains an effective and rapid method of imaging, which in many cases is diagnostic $(1,3,5,6,17)$ and graded as COR Ia LOE: B both in ESC and AHA/ACC guidelines. However, it should be emphasized that up to $30 \%$ of patients with proven IE subsequently are labelled as "possible" due to negative or equivocal results on echocardiography or blood cultures $(20,21)$. The use of transthoracic echocardiography (TTE) in early stage of diagnosis is the method of imaging recommended in the patient with the suspicion of an infectious endocarditis involving either a native valve (NVE) or a prosthetic valve (PVE). In patients with NVE, TTE has a sensitivity between $50 \%$ and $90 \%$, while the specificity reaches $90 \%$. For suspected PVE, the use of TTE has a lower sensitivity, ranging between $40 \%$ and $70 \%$. However, it provides important information in the assessment of ventricular size and on its function. The TTE adds an additional evaluation on hemodynamic severity of valve lesions, diagnosis of anterior prosthetic aorta valve abscesses, which can be difficult to visualize with the use of transoesophageal echocardiography (TEE). The use of
TEE is indicated when the TTE is positive but it was not sufficient to reach a diagnosis of infectious endocarditis, when intracardiac complications are suspected or when cables of an implantable device are present. In patients with a suspected NVE, the TEE has a sensitivity from $90 \%$ to $100 \%$ and specificity of $90 \%$ for the detection of vegetations and is superior to TTE for detection of complications, such as perforations, abscesses, and fistula. In ESC guidelines $(3,5)$ TTE should be considered in patients with suspected IE, even in cases with positive TTE, except in isolated right-sided native valve IE with good quality TTE examination and unequivocal echocardiographic findings (COR IIa; LOE: C).

Concerns relating to precise diagnosis of PVE still persist. In a recent meta-analysis, a reduced aggregate sensitivity of $86 \%$ was shown [95\% confidence interval (CI): from $77 \%$ to $92 \%$ ] for TEE in conferring a certainty for the diagnosis of IE (22). As such, other imaging modalities are emerging to help to make or exclude the diagnosis in cases where TEE is not diagnostic. Even when abnormalities are detected, it can be difficult to differentiate the presence of nodules from small vegetations or to distinguish signs of infection from tissue changes that occurred in the postoperative period. The use of cardiac computed tomography (CT) scanning has established itself as the key additional modality to be used when echocardiography does not provide clearly delineated anatomy information. The CT scanning has earned a recommendation to Class II, LOE: B for use in IE in ACC/AHA 2014 guidelines for heart disease $(1,6)$. Compared to TEE, cardiac CT can be considered equivalent or even superior to TEE to demonstrate precise features of anatomy and paravalvular complications such as paravalvular abscesses or mycotic aneurysms. In addition, it is less prone to artifact compared to echocardiography in the evaluation of prosthetic valves. The use of concomitant CT angiography has become common practice because this approach greatly assists surgical strategy planning. Its wide use allows the exclusion of a significant coronary artery disease in younger patients who can avoid coronary angiography. Detection of para-valvular lesions using CT imaging is now an important diagnostic criterion in the ESC 2015 guidelines (3) on IE despite not yet reaching a precise COR and LOE.

\section{New evidence}

A large consensus for the diagnosis of IE has occurred with the use of combination of CT imaging with metabolic 
imaging by 18 -fluorodeoxyglucose positron emission tomography $\left({ }^{18} \mathrm{FDG}-\mathrm{PET}\right)$ or leukocyte scintigraphy (radiolabeled leukocyte single-photon emission computed tomography (SPECT). This method is very promising because it allows visualisation of regions with metabolic activity or inflammation in patients who, according to the Duke criteria, have "possible" IE or suspected of cardiac device infection. Several reports have demonstrated the effectiveness of this method. One study (23) evaluated the effect of 18 FDG-PET in 72 patients who had suspected PVE reporting diagnosis with an overall sensitivity of $73 \%$ and a specificity of $80 \%$. The patients who were managed with 18FDG-PET as the additional criterion of "abnormal prosthetic valve 18FDG-PET signal" showed an increased sensitivity of the modified Duke criteria from $70 \%$ to $95 \%$ thus reducing the number of patients with "possible IE" from $56 \%$ to $32 \%$. In another report (24), 76 of 92 patients with suspected PVE or CDI were studied using 18FDGPET/CT (angiography), CT and echocardiography providing a conclusive diagnosis in $95 \%$ of cases overall. The addition of this procedure has been associated with greater general sensitivity and specificity ranging between $87 \%$ and $90 \%$, respectively, and an increased sensitivity of the modified Duke criteria from $51 \%$ to $91 \%$.

Leukocyte scintigraphy with SPECT/CT imaging has also been shown to be a valid test with a reported sensitivity of $90 \%$ and a specificity of $100 \%$ in a cohort study including 131 patients (25). Another report showed inconclusive results using the echocardiography in 39 patients with suspected PVE. The results with the use of 18FDG-PET/CT showed a higher sensitivity compared to SPECT/CT images, but the SPECT had a greater specificity (26). The ESC 2015 guidelines emphasized the abnormal meaning of imaging with the use of 18FDGPET /SPECT; a positive sign at the site of a prosthetic valve (if implanted $>3$ months previously) is now considered an important diagnostic criterion for PVE. However, this criterion is not yet included in the AHA/ACC. Relevant studies have underlined the fact that the cross-sectional routine imaging of the brain, chest, spine and viscera can be diagnostic, and can change the IE management. These imaging cohort studies suggest that patients with IE have a high incidence of subclinical complications, such as hemorrhage, embolism, or abscess. In one prospective study based on the images of routine cerebral magnetic resonance (MRI), abnormalities were identified in $80 \%$ of patients and simultaneously 'upgraded' 26\% (14 of 53 patients) diagnosis of IE from "possible" to "defined" (27). In another study, the use of cerebral angiography CT identified the presence of intracranial mycotic aneurysms in $32 \%$ of patients with left side endocarditis. Subsequently $50 \%$ of them had endovascular or neurosurgical procedures (28). Iung et al. (29) revealed anomalies identified in the spleen, liver, or kidneys for $34 \%$ of patients on magnetic resonance imaging of the abdomen. As a result, evidence of embolism diagnosed through the transverse imaging is considered as a minor novel diagnostic criterion in the ESC guidelines 2015 (3).

Since evidence of cross-sectional imaging, be it cardiac CT and 18FDG-PET or SPECT have the potential to improve the diagnosis and detection of complications in patients with suspected IE, it has been incorporated in routine practice for diagnosis in the subset of patients termed "Duke possible "and for CDI. However, potential disadvantages do exist with these modalities. The main drawback is that metabolic imaging cannot be accurate in discriminating between barren inflammation and infection, and is therefore of limited use in the early postoperative period. The literature reports numerous false positives for PET/CT imaging after cardiac surgery due to postpericardiotomy syndrome, prosthetic valve thrombosis and after aortic operation for IE. Two other points to consider include access to the advanced imaging procedures, which is often limited, hence when emergency intervention is required, there is a risk of incurring logistical obstacles that may delay definitive surgery. The second is related to the fact that at the moment we are unaware of any randomized trials that have compared different groups, and it is unlikely that such a trial will be conducted. Certainly, the identification of which groups of patients from the clinical point of view who would benefit the most from advanced imaging and through which mode it should be precisely executed remains undetermined (Figure 2).

\section{Routine follow-up imaging}

It is agreed that early post-operative imaging is recommended in all patients who underwent surgery for IE but the recommendations are incongruent. Followup imaging is recommended at 30 days by $\operatorname{ESC}(3,5,17)$, while 6 weeks to 3 months after valve implantation is recommended by the ACC/AHA (1,6,8-10), although the general orientation is to perform an echocardiography check at the time of hospital discharge. In patients who have received mechanical valves, the guidelines do not recommend follow-up imaging for those who are stable unless there is another indication. In bioprosthetic valve 


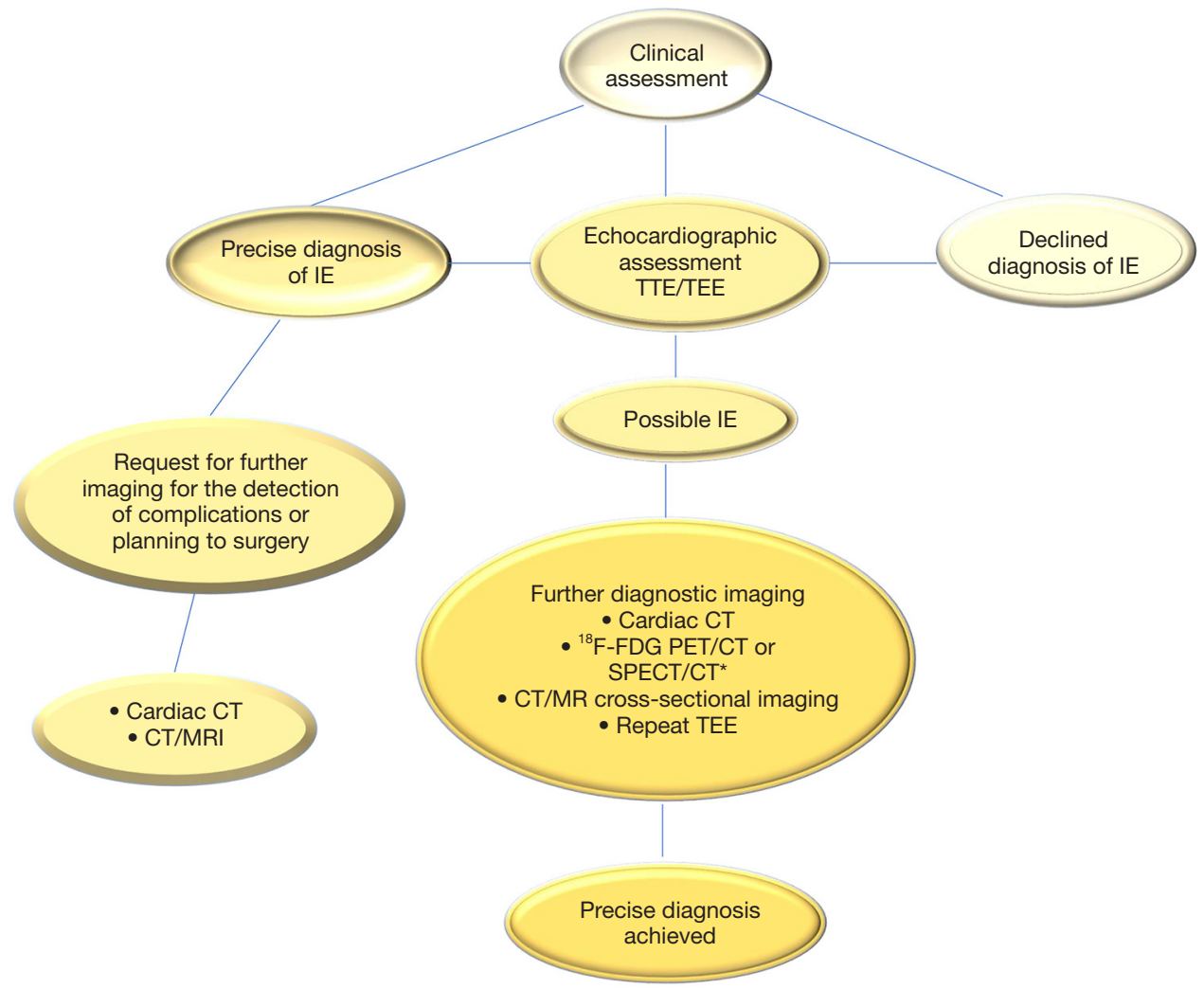

Figure 2 Show the applied strategy of integrated imaging in patients with suspected infective endocarditis (IE). In patients included in the subgroup with possible IE after initial evaluation by TTE and TEE a cardiac CT imaging, metabolic imaging or transverse imaging of the head and viscera by CT scan or MRI is indicated to achieve a precise early diagnosis. For suspected IE a ${ }^{18}$ FDG-PET/CT or a crosssectional imaging by CT or MRI (or metabolic imaging) scans may assist with detection of complications, such as abscess, mycotic aneurysm, infarct, or hemorrhage in patients with definite IE. *, ${ }^{18} \mathrm{~F}$-PDG SPECT/CT is useful in patients with prosthetic valves or cardiac implantable electronic devices. IE, infective endocarditis; CT, computed tomography; ${ }^{18} \mathrm{FDG}$-PET/CT, 18 -fluorodeoxyglucose positron emission; MRI, magnetic resonance imaging; NVE, native valve endocarditis; PVE, prosthetic valve endocarditis; TEE, transoesophageal echocardiography TTE, transthoracic echocardiography; SPECT, single-photon emission computed tomography.

recipients, who are asymptomatic, routine annual TTE follow-up imaging is recommended by ESC guidelines (5), while ACC/AHA guidelines $(1,6)$ recommend annual TTE only after 10 years from the date of implantation of the valve. Both guidelines recommend the TTE for the PHV dysfunction symptoms/signs irrespective of the implant date and in some patients at higher risk of accelerated deterioration of the valve even in the absence of symptoms. The ESC's recommendation is based on a declaration of consent document (30), following the recommendations of the Valve Academic Research Consortium-2 (31). In patients who received conventional stented/non-stented xenograft, the ACC/AHA recommendation is based on observations that the incidence of deterioration of structural valves is low for bioprosthetic valves in the first 10 years after implantation.

\section{Antibiotic therapy}

For the cardiologist and the surgeon, the main challenges for the success of the antibiotic therapy are represented by bacterial tolerance and antibiotic resistance. The persistence of phenotypic variants of bacteria despite antibiotic therapy may be the cause of tolerance. The result sees a resumption of growth and infection once antibiotic concentrations decrease. The mechanisms involved in tolerance are manifold, including the high bacterial density and poor penetration of antibiotics within the vegetations, low 
metabolic bacterial activity and the production of protective biofilm on the prosthetic material (32). The increase in cases of Staphylococcus aureus infection has further enhanced the problem of antibiotic resistance. For this reason, new strategies should be used to prevent and treat IE caused by strains that form multidrug-resistant $S$ aureus biofilm. They are targeted at to the initial bacterial adhesion inhibition to living and inert surfaces, so as to reduce further the development of the biofilm. One other action is targeted at the interruption of the biofilm architecture as well as to anti-pathogenic approaches or signal interference involving the inhibition of quorum sensing (33). The prevention of bacterial adhesion to subsequent insertion of the intracardiac device deserves particular attention. It is crucial and can be obtained by using implants coated with various adhesion inhibitors. Despite antibiotics, ionic silver, and silver nanoparticle exerting inhibitory actions on biofilm formation, coated implants were ineffective and poorly tolerated in humans. The use of monoclonal antibodies such as TRL1068 has shown some promise with regards to interrupting the biofilm architecture and is currently under evaluation. In an in vivo mouse model a biofilm was created through a resistant methicillin $S$ aureus infection which was subsequently treated using a combination of TRL1068 with daptomycin. The association of the two products has significantly reduced the adherent bacteria counts compared to daptomycin alone (34).

Prolonged antibiotic therapy may lead to toxicity and side effects to the patients. Therefore, it is necessary to balance the effectiveness of treatment with the overall risk due to prolonged antibiotic administration. Emerging evidence in support of short-term antibiotic treatment is gradually directed at selected patients. In patients with simple IE caused by oral streptococci in which renal function is preserved, a combination of a penicillin or ceftriaxone with an aminoglycoside for a total of 14 days is indicated and has been proven to be safe and effective (35). Likewise, a 2 -week monotherapy cycle using penicillin or penicillinaminoglycoside in combination demonstrated efficacy in patients with right-sided IE who were not sensitive to methicillin (36). The use of aminoglycosides, as growing data suggest, however, may cause harm without clear clinical benefits. Fowler et al. (37) in an RCT, compared the use of daptomycin to conventional therapy (penicillin or vancomycin with initial gentamicin) in patients with right side endocarditis sustained by $S$ aureus bacteremia. Daptomycin has been shown to be non-inferior. The authors highlighted the occurrence of renal dysfunction in $11 \%$ of patients in the group treated with daptomycin compared to $26 \%$ of the group that received conventional therapy $(37,38)$.

The use of aminoglycosides has reduced and they were removed from guidelines of the AHA/ACC (1,6,8-10) and ESC $(3,5,17)$ in patients with methicillin-resistant or methicillin sensitive $S$ aureus NVE. Although aminoglycosides have gained wide acceptance with extensive use in the treatment of enterococci IE; however, the increased frequency of resistance (from 25\% to $50 \%$ of isolates in recent studies), alongside the recognition of the potential harm, prompted the ESC 2015 steering committee to identify ampicillin and ceftriaxone (class IB recommendation) as a treatment of choice for Enterococcus faecalis resistant to aminoglycosides. There are substantial observational studies in support of this direction that led to a change in recommendation by the AHA/ACC and ESC. In these reports it was shown that ampicillin/ceftriaxone was just as effective with reduced levels of nephrotoxicity $(39,40)$. Although, current AHA/ACC guidelines (41) suggest that the remaining duration of antibiotics should be administered (including administration prior to surgery) based on level C evidence. However, rationalizing treatment with antibiotics in patients who have undergone successful surgery and have negative valve cultures can be considered. In these patients, the evidence suggests 2 weeks of antibiotic therapy may be sufficient for eliminating the qualifying pathogen with a margin of safety $(42,43)$ (Figures 3,4$)$.

\section{New evidence}

A benefit towards a rapid transition to oral regimens of antibiotics with good bioavailability can facilitate a reduction in hospital stay. Only 1 published RCT POET (Partial Oral Treatment of Endocarditis) (44) that recruited 400 patients who had endocarditis on the left side of the heart caused by streptococcus, Enterococcus faecalis, Staphylococcus aureus, or coagulase-negative staphylococci compared outcomes between intravenous antibiotics to continue intravenous treatment (199 patients) or an oral antibiotic treatment switch (201 patients). The primary outcome was a composite of all-cause mortality, unplanned cardiac surgery, embolic events, or relapse of bacteremia with the primary pathogen, from the time of randomization until 6 months after antibiotic treatment was completed. The primary composite outcome was $12.1 \%$ in the patients who received intravenously treatment and $9.0 \%$ in those who were managed with the orally administration of 


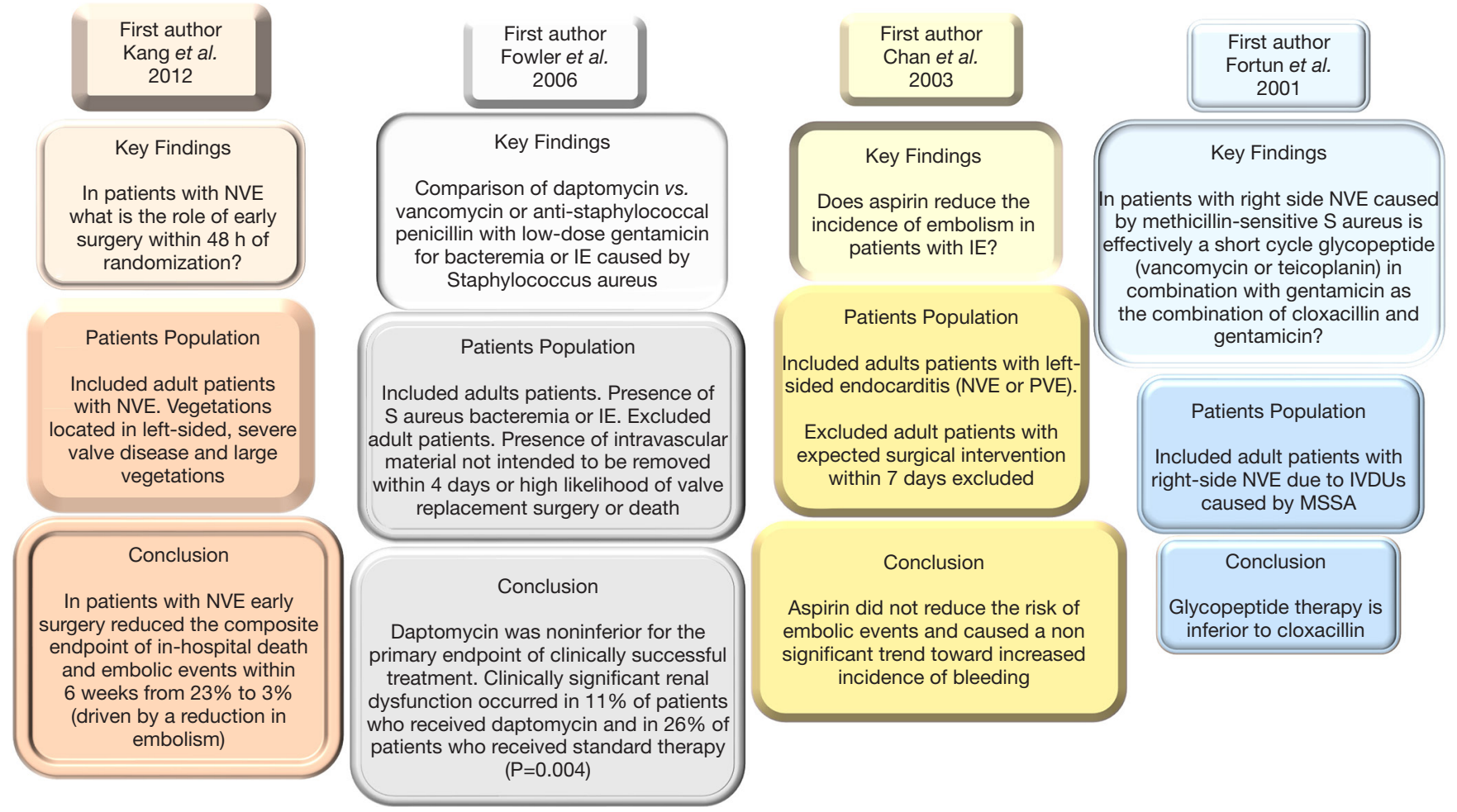

Figure 3 Includes 20 yrs 4 RCT of IE published from 2001 to 2012. IE, infective endocarditis; IVDU, intravenous drug user; MSSA, methicillin-sensitive Staphylococcus aureus; NVE, native valve endocarditis; PVE, prosthetic valve endocarditis; RCT, randomized controlled trial.

antibiotics (3.1 percentage points; $95 \%$ confidence interval, -3.4 to $9.6 ; \mathrm{P}=0.40$ ) showing that oral antibiotic treatment was non-inferior to continued intravenous antibiotic treatment (44).

The benefits associated with the use of outpatient parenteral antibiotic therapy (OPAT) can facilitate early hospital discharge. OPAT may be initiated in specific patients after completion of the first 2 weeks of treatment because the risk of complications is reduced. OPAT is contraindicated in patients who have heart failure, complicated infection, high risk of embolism, neurological complications or renal insufficiency (45-47). Early hospital discharge is currently being investigated in the ongoing RCT OPAT (Outpatient Parenteral Antimicrobial Therapy) with an estimated study completion date of December 31, 2021. The purpose of this study is to investigate the efficacy, safety and cost effectiveness of treatments administered at the new OPAT unit of the University Hospital of Lausanne within the Swiss Health System. The OPAT study recruited 2,000 patients. The primary end point is the efficacy classified 3 months after the end of treatment as the absence of fever and no clinical signs of site infection, readmission to hospital due to complications related to the infection and absence of recurrence of the infection within 3 months post-OPAT.

Finally, the use of ciprofloxacin and rifampicin has been evaluated in IV drug users (IVDU) with Methicillinresistant $S$ aureus NVE. The RCT data demonstrated safety and efficacy after oral administration of antibiotics, although an increase in rates of resistance to fluoroquinolone limited its applicability (48).

\section{Stroke and IE}

Stroke occurs as a complication in $20-50 \%$ of cases in patients with IE $(1-3,5)$. The onset of stroke is a serious event because besides causing variable disability, it is an independent adverse prognostic factor for survival $(49,50)$. Different studies suggest that the risk of stroke is higher at diagnosis and decreases rapidly after the use of antibiotic 

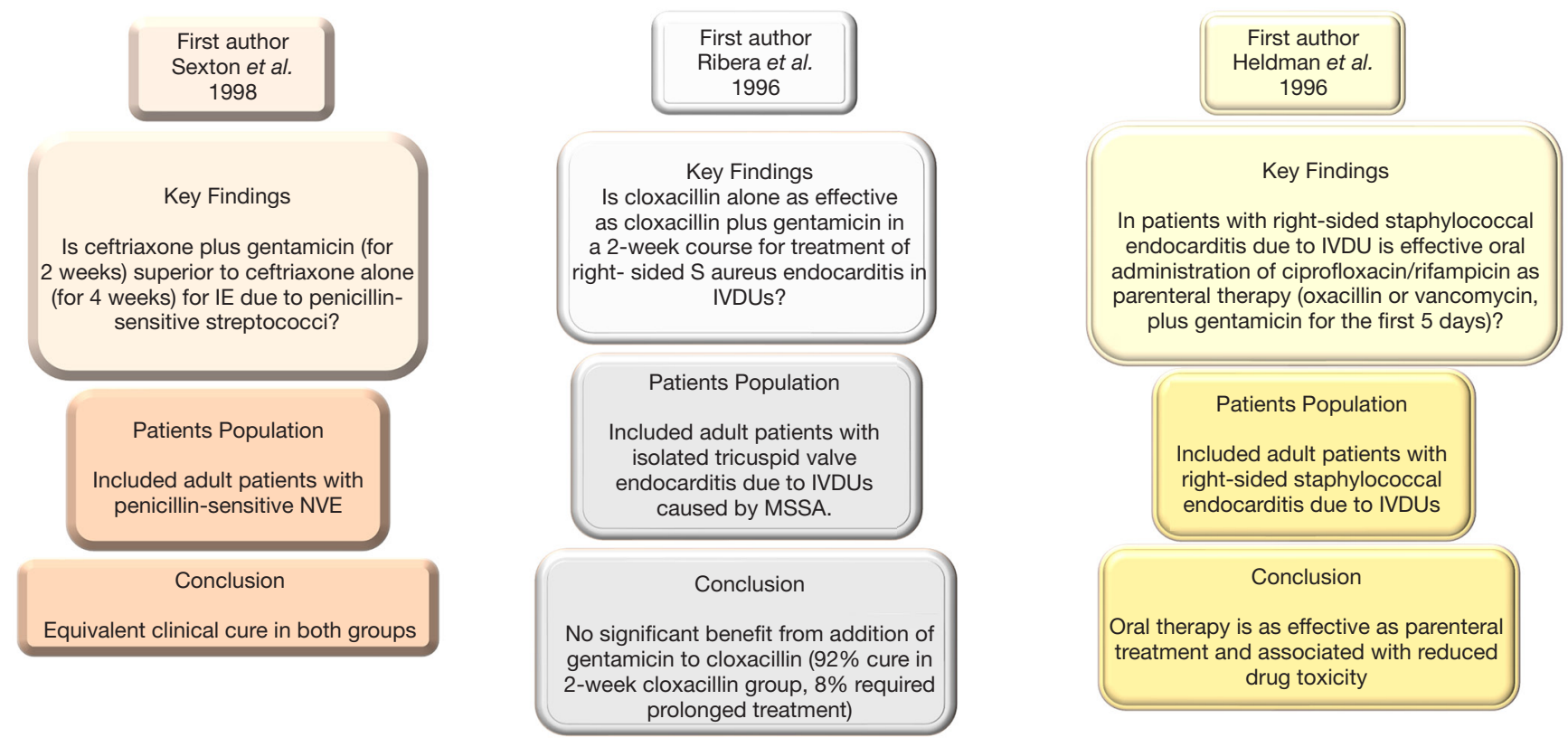

Figure 4 Includes 20 yrs 3 RCT of IE published from 1996 to 1998. IVDU, intravenous drug user; MSSA, methicillin-sensitive Staphylococcus aureus; RCT, randomized controlled trial.

therapy in the initial phase of infection. Dickerman et al. (51) reported that the incidence of neurological complication drops from 4.82 per 1,000 patient days in the first week of therapy to 1.71 per 1,000 patient days in the second week. Risk factors identified for embolism are the size of the vegetation $(>10-15 \mathrm{~mm})$, the involvement of the mitral valve, the mobility of the vegetation and $S$ aureus infection.

We have shown that the serious pathological damage that often occurs in cauliflower-like friable vegetations of valve leaflets, with or without the extension of the lesion to the annulus, is an independent factor for embolic stroke. However, the key unresolved challenge in the contemporary management of IE, keeping the debate alive in multidisciplinary heart team decision making, is the role of surgery in the prevention of stroke/embolism, and patient selection for choosing the optimal time to surgery. In the 2015 update of the AHA/ACC guidelines, surgery is recommended with an indication as Class IIa for surgery to prevent recurrent embolism in patients with previous emboli greater $\geq 10 \mathrm{~mm}$ and with high risk of further embolism. The risk is defined as persistent or enlarged vegetation $(17,41)$. Similarly for the ESC guidelines the surgery is categorized as a Class I recommendation, useful in preventing recurrent emboli in patients with persistent vegetation $>10 \mathrm{~mm}$ in size (3). Surgery is indicated as a Class IIa recommendation in patients at risk of first embolism (vegetation $>10 \mathrm{~mm}$ in size) if associated with severe valve regurgitation or stenosis. This AHA/ACC and ESC COR and LOE degree is based on RCT evidence (52). In patients at high risk of embolism/stroke due to the presence of vegetations greater than $15 \mathrm{~mm}$, despite the absence of valve dysfunction, surgery for the prevention of embolism can be considered. This choice, however, is rarely undertaken as the sole indication. In patients who have already had a stroke the optimal timing of surgery remains a matter of debate, because it is based on a number of older studies that have reported poor results in patients who underwent early surgery (53). For these, in fact, there is a risk of haemorrhagic transformation caused by anticoagulation during cardiopulmonary bypass, as well as by hypotension that can intervene during surgery. Both of these variables can be the cause of theoretical worsening of cerebral ischemia. Unfortunately, definitive conclusions cannot be given due to the fact that observational studies directed towards this topic have generally been small, inadequately controlled $(49,54)$ and based on unmatched confounders related to the unmeasurable judgment of the operating surgeons (2,55-62) (Figures 5,6). 


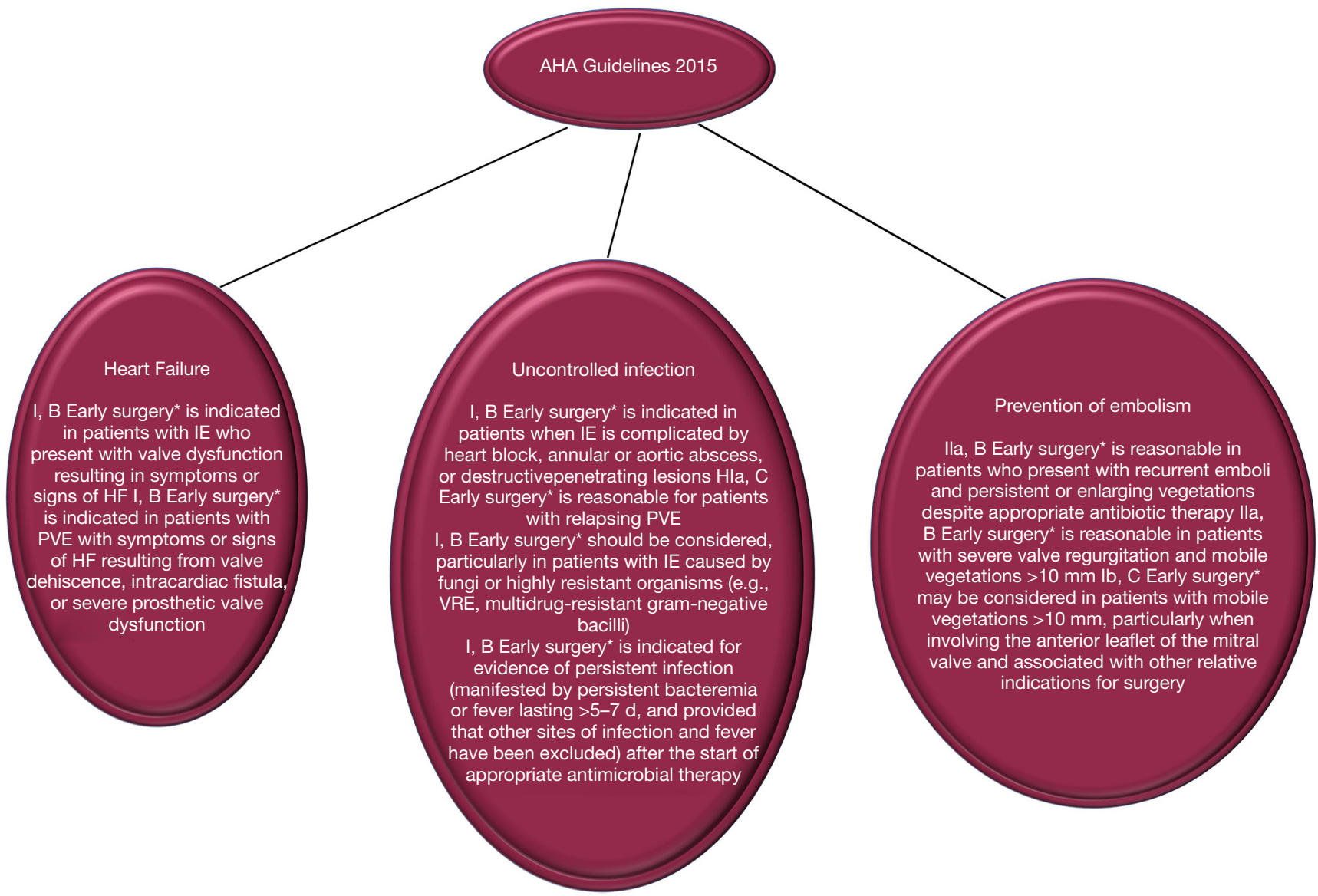

Figure 5 Indications for surgery in AHA. *, defined as "during initial hospitalization and before completion of a full course of antibiotics." HF, heart failure; NVE, native valve infective endocarditis; PVE, prosthetic valve infective endocarditis; VRE, vancomycin-resistant Enterococcus.

\section{New evidence}

ICE-PCS collaboration (54) reported the largest series of 867 patients with IE complicated by ischemic stroke syndromes and 198 of these who underwent valve replacement surgery post-stroke. The study evaluated the effect of 1-year mortality in 58 patients who had early surgery compared to those $(\mathrm{n}=147)$ who received a later operation. The 58 patients undergoing surgery within 7 days after diagnosis had a trend towards increased inhospital mortality rates than those whose surgery was managed later $(27.1 \%$ in early surgery and $19.2 \%$ in late surgery group, $\mathrm{P}=0.328$; adjusted hazard ratio, $1.138 ; 95 \%$ CI, 0.802-1.650). Based on the interpretation of this result, the guidelines of AHA/ACC $(1,6,41)$ and $\operatorname{ESC}(3,5,17)$ suggest surgery as an option to be considered if safe, wherever necessary, although stroke remains a common reason for avoiding surgery in everyday practice (63). On the contrary, in the interpretation of the guidelines based on various studies, the transient ischemic attack or silent embolism should not delay the indicated surgery for other reasons (49). This includes instances when the presence of particularly insidious pathogens for which the diagnosis can be too late (60). In contrast, patients with cerebral hemorrhage or complex stroke resulting in a coma have a significantly higher surgical mortality and surgery should be postponed for at least 4 weeks if the multidisciplinary heart team indicates surgery in these patients $(63,64)$. Finally, the management of patients with minor bleeding or mild hemorrhagic conversion of an ischemic stroke deserves consideration. For this category of patients, the clinical scenario can often be complex and the risk/benefit equation often challenges any rigid recommendations. 


\section{Surgery}

The specific indications for performing surgery in case of HVE are the presence of progressive damage to the valve and tissues, uncontrolled infection and high risk of embolism. Therefore, the first objective of surgery is to remove the infected tissue and/or the presence of foreign material; to abolish the threat of embolism sources; to completely debride the infected paravalvular tissue and any cavities that are formed; restore cardiac integrity and valve function. Although the use of various surgical techniques have been chosen, for example the repair of the mitral valve or aortic allograft implantation, however, the results have not yet demonstrated a clear long-term advantage of one technique over the other. In patients requiring surgery for an HVE, regardless of which approach is chosen, evidence has shown that long-term outcomes are inferior than elective valve surgery. In fact, 10 -year survival varies from $40 \%$ to $60 \%(6,65,66)$ and the choice of the ideal substitute for HVE surgery remains an open debate $(2,55-62,67)$. It is not clear whether this delay in mortality refers to late complications of prosthetic valve, extracardiac manifestations of the disease, or relapse of infection due to the persistence of the complex biofilm of the pathogen responsible for the HVE. In patients with HVE, the use of surgery is currently performed from $50 \%$ to $60 \%$ with reported survival rates at 6 months which are $>80 \%(53,63)$. The indications for surgery were mainly based on the benefits, reported in observational and cohort studies, and highlighted in patients with valve dysfunction that causes heart failure, uncontrolled infection or recurrent embolism. The uncontrolled infection is the condition in which the infection has had a paravalvular extension, development of abscess, or persistent bacteremia. The latter, if manifested in a particular patient, can be reason for discussion in the heart team shared decision making, when there is mild or relative heart failure (68). Figures 2 and 3 show the indications for surgery in accordance with the guidelines of the AHA/ ACC and ESC. It is interesting to note that in real world situations although there is a significant number of patients who have a clear indication for surgery in accordance with the guidelines, surgery is still not performed. In a study by the ICE-PCS (International Collaboration on EndocarditisPotential Cohort Study) registry, this significant percentage of patients with left-sided IE, for whom surgical treatment is required, reaches $24 \%$ (202 of 863) (63). As it emerges from the ICE-PCS registry, many predictive factors influence the choice to avoid surgery. The avoidance of surgery despite HVE was dictated by the presence of liver disease [odds ratio (OR) for surgery: 0.16 ; $95 \%$ CI: 0.04 to 0.64], infection determined by $S$ aureus (OR: $0.50 ; 95 \%$ CI: 0.30 to 0.85 ) and stroke before decision to perform surgery (OR: 0.54 ; 95\% CI: 0.32 to 0.90 ). Instead the presence of severe aortic regurgitation, abscess and embolization was an indication for surgical choice. For 181 patients who avoided a surgical procedure, the reasons included an unfavorable early prognosis regardless of treatment (34\%), hemodynamic instability (20\%), death before surgery (23\%), stroke (23\%), sepsis (21\%) and surgeon's refusal to operate (26\%). Ultimately, in the study of ICE-PCS group, the perceived risk of the operation determines the threshold for surgical treatment. Surgical procedures for active IE present a high risk, with an overall hospital mortality of $20 \%$ which in many centers can be even higher (63).

\section{New evidence}

An important determinant in the choice of surgery in case of HVE would come from the improvement of the risk assessment models for IE in order to better clarify the decision-making process.

The Society of Thoracic Surgeons database was used to derive a surgical risk score in patients with IE who were likely to have surgery by Gaca et al. (69). The model identified 13 risk factors for mortality, including emergency status, cardiogenic shock, hemodialysis and active endocarditis. The De Feo et al. (70) and MartínezSellés et al. (71) studies incorporated multiple detailed parameters of infection, including valve type and organism. The PALSUSE score included patients aged $\geq 70$ years and the strong extension of the focus of infection with substantial intracardiac destruction as risk factors. Other factors in PALSUSE are as follows: staphylococcus infection, urgent surgery, female gender and EuroSCORE (European System for Cardiac Operative Risk Assessment) value of $\geq 10$ as predictors of mortality in hospital, with inhospital mortality ranging from $0 \%$ in patients with a score of 0 , to $45 \%$ in patients with a score $>3$ (71). The optimal timing of surgery remains a controversial cause for debate. Advantages and disadvantages are worth considering. On the one hand, delaying surgery may allow for a longer duration of antibiotic therapy associated with better hemodynamic stabilization. Conversely, the postponement of the surgical procedure can result in the risk of disease progression with the valve destruction, abscess formation, heart block, embolic complications and even death. In fact, 


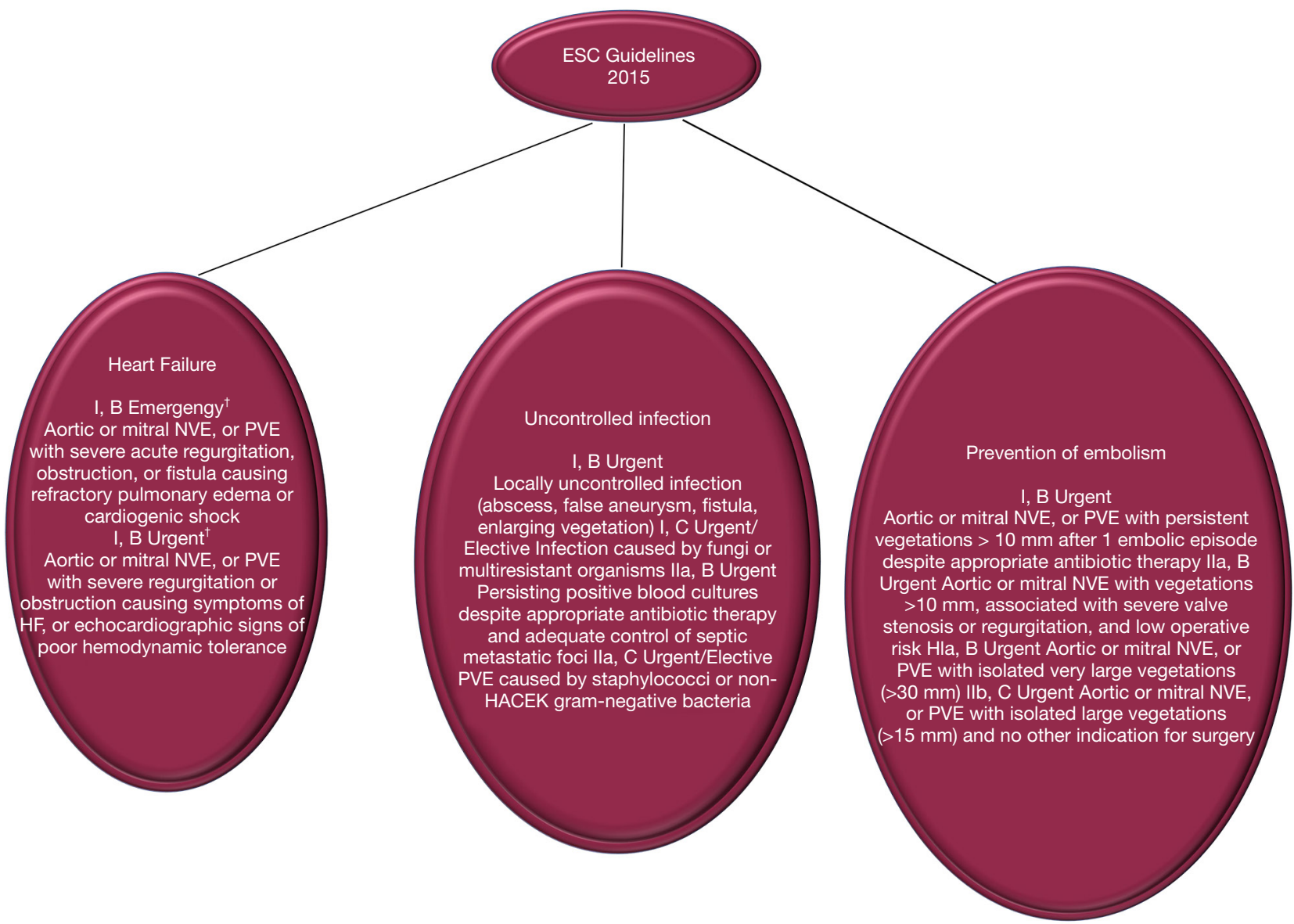

Figure 6 Indications for surgery in ESC. ${ }^{\dagger}$, defined as: emergency surgery = performed within 24 h; urgent surgery = within a few days; elective surgery $=$ after at least 1 to 2 weeks of antibiotic therapy. HACEK, Haemophilus species, Aggregatibacter species, Cardiobacterium hominis, Eikenella corrodens, and Kingella species; HF, heart failure; NVE, native valve infective endocarditis; PVE, prosthetic valve infective endocarditis; VRE, vancomycin-resistant Enterococcus.

for some outcomes such as embolism, the potential gains of the surgery are reduced over time (51). In 2012, in a pivotal Korean RCT (52), HVE surgery was randomized to early surgery (performed within 48 hours of randomization) and conventional care in patients with NVE, severe valve regurgitation and large vegetations. The patient population included in the study reported by Kang et al. (52) were young (average age 47 years), with few comorbidities and predominantly streptococcal infection. In the results it was shown that early surgery was associated with a significant reduction in the composite endpoint of death or hospital embolism. In addition, surgery was required for more than $90 \%$ of patients receiving conventional care, thus validating indications for surgery. This study is a point of reference for the strategy to be applied in HVE and has encouraged a trend towards early surgery. However, the results found in the study are limited and its applicability is uncertain in older populations with multiple comorbidities and staphylococcal infection. The findings of the Korean study (52) conflict with the results of the studies from the ICE-PCS registry (51), which define early surgery as being undertaken "during the initial hospitalization for IE”. Although early surgery for patients with NVE was associated with reduced mortality, this was not true for patients with PVE after adjusting for confounding variables $(55,57)$.

Cardiac surgeons are faced with a different interpretation of the emergency/urgency criterion expressed in the international guidelines. The emphasis on "early surgery" differs significantly between European and US 
guidelines. The ESC guidelines $(3,5,17)$ distinguishes emergency surgery (performed within 24 hours), urgent surgery (within a few days) and elective surgery (after $1-2$ weeks of antibiotic therapy), restricting to patients in whom urgent surgery is recommended for most cases. On the contrary, the AHA guidelines $(1,6,8,9,41)$ define early surgery as "during the initial hospitalization and before completion of a full course of antibiotics." Based on our experience and considering many reports we can conclude that there are no proven benefits in delaying surgery once an indication for surgery has been established. The choice of timing for the surgery between the same day of hospitalization/diagnosis or 48 hours from hospitalization for HVE depends on the individual clinical circumstance and availability of adequate surgical skills $(2,55-62,67,72)$. The current series have shown that it is possible to obtain very low mortality in centers of excellence with high level experience in managing complex patients where the different skills bring together experts in cardiology, microbiology and surgery (60). The controversy that emerges in the management of the patient with HVE that requires the choice of early surgery can only be resolved through solid tests that advance the field. RCT level data is needed to guide change of practice because data from observational studies may lack robustness. Based on these, it is more difficult to progress because there is concern that observational/cohort studies may be biased in favor of conventional mechanical or stented xenograft prosthesis by unmatched confounders related to the personal consideration of the engaging surgeons (55-59). As can be seen from Figure 4 in the last 20 years, only 7 randomized studies involving IE patients have been published, most of primarily focused on antibiotic therapy and only one on the optimal time for the surgical procedure (52).

The solution to this conundrum would be to carefully define the priorities for new RCTs. They must be reasonable and acceptable to the medical community. Multicenter studies are demanding and may be limited to concerns regarding becoming outliers as experience and results vary widely between centers. However, the advantage of multicenter registry studies is in the very large volume of patients participating, as few centers have the volume to perform these studies individually. Furthermore, unresolved issues, such as early surgery, may be side-lined due to competing/divergent research. This is clearly evident in patients with PVE. Should they be clustered with the other patients in indications for surgery? Is it recommended that all patients with IE and severe valve dysfunction undergo surgery, even if they do not have heart failure? Finally, as proposed by San Román et al. (68), a randomized clinical trial of patients with left-sided IE and high-risk characteristics, but without classic surgical indications, would be useful. Enrolment groups should include those receiving surgery within 48 hours or patients destined for conventional care, with mortality as the primary endpoint. Although logistically demanding, this study would not only be of widespread interest but would be valuable and could announce the long-awaited transition from observational studies to RCT-level research (56-59).

\section{Conclusions}

We have noted several differences between guidelines of the AHA/ACC recommendations regarding the management of patients with HVE. The existence of non-binding recommendations on guidelines seem contradictory, especially if indicative for important decisions such as the optimal timing of surgical treatment. We have also found that a small number of observational studies were cited by both guidelines, although supporting opposite recommendations. The lack of high-quality evidence for the management of patients with HVE is not only obvious but is restricted to the management of patients who are often in severe clinical conditions. The short-term goal would be to increase randomized trials for these patients because in their absence, questions regarding the validity of these recommendations will remain topics of discussion.

\section{Acknowledgments}

Funding: None.

\section{Footnote}

Provenance and Peer Review: This article was commissioned by the Guest Editors (Drs. Francesco Nappi, Christos Mihos, and Cristiano Spadaccio) for the series "Infective Endocarditis in the 21st Century" published in Annals of Translational Medicine. The article was sent for external peer review organized by the Guest Editors and the editorial office.

Reporting Checklist: The authors have completed the Narrative Review reporting checklist. Available at http:// dx.doi.org/10.21037/atm-20-3739 
Conflicts of Interest: The authors have completed the ICMJE uniform disclosure form (available at http:// dx.doi.org/10.21037/atm-20-3739). The series "Infective Endocarditis in the 21st Century" was commissioned by the editorial office without any funding or sponsorship. FN served as the unpaid Guest Editor of the series and serves as an unpaid editorial board member of Annals of Translational Medicine from Feb 2019 to Jan 2021. CS served as the unpaid Guest Editor of the series. Dr. MRM reports that he's the member of Medtronic - Scientific Advisory Board. The authors have no other conflicts of interest to declare.

Ethical Statement: The authors are accountable for all aspects of the work in ensuring that questions related to the accuracy or integrity of any part of the work are appropriately investigated and resolved.

Open Access Statement: This is an Open Access article distributed in accordance with the Creative Commons Attribution-NonCommercial-NoDerivs 4.0 International License (CC BY-NC-ND 4.0), which permits the noncommercial replication and distribution of the article with the strict proviso that no changes or edits are made and the original work is properly cited (including links to both the formal publication through the relevant DOI and the license). See: https://creativecommons.org/licenses/by-nc-nd/4.0/.

\section{References}

1. Nishimura RA, Otto CM, Bonow RO, et al. 2014 AHA/ ACC guideline for the management of patients with valvular heart disease: a report of the American College of Cardiology/American Heart Association Task Force on Practice Guidelines. Circulation 2014;129:e521-643.

2. Savage EB, Saha-Chaudhuri P, Asher CR, et al. Outcomes and prosthesis choice for active aortic valve infective endocarditis: analysis of the Society of Thoracic Surgeons Adult Cardiac Surgery Database. Ann Thorac Surg 2014;98:806-14.

3. Habib G, Lancellotti P, Antunes MJ, et al. 2015 ESC guidelines for the management of infective endocarditis. Eur Heart J 2015;36:3075-128.

4. Pettersson GB, Coselli JS, Hussain ST, et al. 2016 The American Association for Thoracic Surgery (AATS) consensus guidelines: Surgical treatment of infective endocarditis: Executive summary. J Thorac Cardiovasc Surg 2017;153:1241-1258.e29.

5. Baumgartner H, Falk V, Bax JJ, et al.; ESC Scientific
Document Group. Guidelines for the management of valvular heart disease. Eur Heart J 2017;38:2739-91.

6. Nishimura RA, Otto CM, Bonow RO, et al. 2017 AHA/ ACC Focused Update of the 2014 AHA/ACC Guideline for the Management of Patients with Valvular Heart Disease: A Report of the American College of Cardiology/ American Heart Association Task Force on Clinical Practice Guidelines. Circulation 2017;135:e1159-e1195.

7. Wilson W, Taubert KA, Gewitz M, et al. Prevention of infective endocarditis guidelines from the American Heart Association: guidelines from the American Heart Association Rheumatic Fever, Endocarditis, and Kawasaki Disease Committee, Council on Cardiovascular Disease in the Young, and the Council on Clinical Cardiology, Council on Cardiovascular Surgery and Anesthesia, and the Quality of Care and Outcomes Research Interdisciplinary Working Group. Circulation 2007;116:1736-54.

8. Desimone DC, Tleyjeh IM, Correa de Sa DD, et al. Incidence of infective endocarditis caused by viridans group streptococci before and after publication of the 2007 American Heart Association's endocarditis prevention guidelines. Circulation 2012;126:60-4.

9. DeSimone DC, Tleyjeh IM, Correa de Sa DD, et al. Incidence of infective endocarditis due to viridans group streptococci before and after the 2007 American Heart Association's prevention guidelines: an extended evaluation of the Olmsted County, Minnesota, population and Nationwide Inpatient Sample. Mayo Clin Proc 2015;90:874-81.

10. Mackie AS, Liu W, Savu A, et al. Infective endocarditis hospitalizations before and after the 2007 American Heart Association prophylaxis guidelines. Can J Cardiol 2016;32:942-8.

11. Bikdeli B, Wang Y, Kim N, et al. Trends in hospitalization rates and outcomes of endocarditis among Medicare beneficiaries. J Am Coll Cardiol 2013;62:2217-26.

12. Pant S, Patel NJ, Deshmukh A, et al. Trends in infective endocarditis incidence, microbiology, and valve replacement in the United States from 2000 to 2011. J Am Coll Cardiol 2015;65:2070-6.

13. Thornhill MH, Dayer MJ, Forde JM, et al. Impact of the NICE guideline recommending cessation of antibiotic prophylaxis for prevention of infective endocarditis: before and after study. BMJ 2011;342:d2392.

14. Dayer MJ, Jones S, Prendergast B, et al. Incidence of infective endocarditis in England, 2000-13: a secular trend, interrupted time-series analysis. Lancet 2015;385:1219-28.

15. Danchin N, Duval X, Leport C. Prophylaxis of infective 
endocarditis: French recommendations 2002. Heart 2005;91:715-8.

16. Duval X, Delahaye F, Alla F, et al. Temporal trends in infective endocarditis in the context of prophylaxis guideline modifications: three successive population-based surveys. J Am Coll Cardiol 2012;59:1968-76.

17. Habib G, Badano L, Tribouilloy C, et al. Guidelines on the prevention, diagnosis, and treatment of infective endocarditis (new version 2009). Endorsed by the European Society of Clinical Microbiology and Infectious Diseases (ESCMID) and by the International Society of Chemotherapy (ISC) for Infection and Cancer. Eur Heart J 2009;30:2369-413.

18. Ruttmann E, Bonatti H, Legit C, et al. Severe endocarditis in transplant recipients-an epidemiologic study. Transpl Int 2005;18:690-6.

19. Pulvirenti JJ, Kerns E, Benson C, et al. Infective endocarditis in injection drug users: importance of human immunodeficiency virus serostatus and degree of immunosuppression. Clin Infect Dis 1996;22:40-5.

20. Vieira ML, Grinberg M, Pomerantzeff PM, et al. Repeated echocardiographic examinations of patients with suspected infective endocarditis. Heart 2004;90:1020-4.

21. Habib G, Derumeaux G, Avierinos JF, et al. Value and limitations of the Duke criteria for the diagnosis of infective endocarditis. J Am Coll Cardiol 1999;33:2023-9.

22. Habets J, Tanis W, Reitsma JB, et al. Are novel noninvasive imaging techniques needed in patients with suspected prosthetic heart valve endocarditis? A systematic review and metaanalysis. Eur Radiol 2015;25:2125-33.

23. Saby L, Laas O, Habib G, et al. Positron emission tomography/computed tomography for diagnosis of prosthetic valve endocarditis: increased valvular $18 \mathrm{~F}$-fluorodeoxyglucose uptake as a novel major criterion. J Am Coll Cardiol 2013;61:2374-82.

24. Pizzi MN, Roque A, Fernández-Hidalgo N, et al. Improving the diagnosis of infective endocarditis in prosthetic valves and intracardiac devices with 18F-fluorodeoxyglucose positron emission tomography/ computed tomography: initial results at an infective endocarditis referral center. Circulation 2015;132:1113-26.

25. Erba PA, Conti U, Lazzeri E, et al. Added value of 99mTc-HMPAO-labeled leukocyte SPECT/CT in the characterization and management of patients with infectious endocarditis. J Nucl Med 2012;53:1235-43.

26. Rouzet F, Chequer R, Benali K, et al. Respective performance of $18 \mathrm{~F}-\mathrm{FDG}$ PET and radiolabeled leukocyte scintigraphy for the diagnosis of prosthetic valve endocarditis. J Nucl Med 2014;55:1980-5.

27. Duval X, Iung B, Klein I, et al. Acute Phase of Endocarditis Study Group. Effect of early cerebral magnetic resonance imaging on clinical decisions in infective endocarditis: a prospective study. Ann Intern Med 2010;152:497-504.

28. Meshaal MS, Kassem HH, Samir A, et al. Impact of routine cerebral CT angiography on treatment decisions in infective endocarditis. PLoS One 2015;10:e0118616.

29. Iung B, Klein I, Mourvillier B, et al. Respective effects of early cerebral and abdominal magnetic resonance imaging on clinical decisions in infective endocarditis. Eur Heart J Cardiovasc Imaging 2012;13:703-10.

30. Capodanno D, Petronio AS, Prendergast B, et al. Standardized definitions of structural deteriorationand valve failure in assessing long-term durability of transcatheter and surgical aortic bioprosthetic valves: a consensus statement from the European Association of Percutaneous Cardiovascular Interventions (EAPCI) endorsed by the European Society of Cardiology (ESC) and the European Association for Cardiothoracic Surgery (EACTS). Eur Heart J 2017;38:3382-90.

31. Kappetein AP, Head SJ, Généreux P, et al. Updated standardized endpoint definitions for transcatheter aortic valve implantation: the Valve Academic Research Consortium-2 consensus document. Eur Heart J 2012;33:2403-18.

32. Lewis K. Platforms for antibiotic discovery. Nat Rev Drug Discovery 2013;12:371-87.

33. Chung PY, Toh YS. Anti-biofilm agents: recent breakthrough against multi-drug resistant Staphylococcus aureus. Pathog Dis 2014;70:231-9.

34. Estellés A, Woischnig AK, Liu K, et al. A high affinity native human antibody disrupts biofilm from Staphylococcus aureus bacteria and potentiates antibiotic efficacy in a mouse implant infection model. Antimicrob Agents Chemother 2016;60:2292-301.

35. Francioli P, Ruch W, Stamboulian D. Treatment of streptococcal endocarditis with a single daily dose of ceftriaxone and netilmicin for 14 days: a prospective multicenter study. Clin Infect Dis 1995;21:1406-10.

36. Ribera E, Gómez-Jimenez J, Cortes E, et al. Effectiveness of cloxacillin with and without gentamicin in shortterm therapy for right-sided Staphylococcus aureus endocarditis. A randomized, controlled trial. Ann Intern Med 1996;125:969-74.

37. Fowler VG Jr, Boucher HW, Corey GR, et al. Daptomycin versus standard therapy for bacteremia and endocarditis 
caused by Staphylococcus aureus. N Engl J Med 2006;355:653-65.

38. Cosgrove SE, Vigliani GA, Campion M, et al. Initial low-dose gentamicin for Staphylococcus aureus bacteremia and endocarditis is nephrotoxic. Clin Infect Dis 2009;48:713-21.

39. Fernández-Hidalgo N, Almirante B, Gavaldà J, et al. Ampicillin plus ceftriaxone is as effective as ampicillin plus gentamicin for treating Enterococcus faecalis infective endocarditis. Clin Infect Dis 2013;56:1261-8.

40. Gavaldà J, Len O, Miró JM, et al. Brief communication: treatment of Enterococcus faecalis endocarditis with ampicillin plus ceftriaxone. Ann Intern Med 2007;146:574-9.

41. Baddour LM, Wilson WR, Bayer AS, et al., American Heart Association Committee on Rheumatic Fever, Endocarditis, and Kawasaki Disease of the Council on Cardiovascular Disease in the Young, Council on Clinical Cardiology, Council on Cardiovascular Surgery and Anesthesia, and Stroke Council. Infective endocarditis in adults: diagnosis, antimicrobial therapy, and management of complications. Circulation 2015;132:1435-86.

42. Morris AJ, Drinkovic D, Pottumarthy S, et al. Bacteriological outcome after valve surgery for active infective endocarditis: implications for duration of treatment after surgery. Clin Infect Dis 2005;41:187-94.

43. Muñoz P, Giannella M, Scoti F, et al. Two weeks of postsurgical therapy may be enough for high-risk cases of endocarditis caused by Streptococcus viridans or Streptococcus bovis. Clin Microbiol Infect 2012;18:293-9.

44. Iversen K, Høst N, Bruun NE, et al. Partial oral treatment of endocarditis. Am Heart J 2013;165:116-22.

45. Andrews MM, von Reyn CF. Patient selection criteria and management guidelines for outpatient parenteral antibiotic therapy for native valve infective endocarditis. Clin Infect Dis 2001;33:203-9.

46. Cervera C, del Río A, García L, et al., Hospital Clinic Endocarditis Study Group. Efficacy and safety of outpatient parenteral antibiotic therapy for infective endocarditis: a ten-year prospective study. Enfermedades Infecciosas y Microbiología Clínica 2011;29:587-92.

47. Duncan CJ, Barr DA, Ho A, et al. Risk factors for failure of outpatient parenteral antibiotic therapy (OPAT) in infective endocarditis. J Antimicrob Chemother 2013;68:1650-4.

48. Heldman AW, Hartert TV, Ray SC, et al. Oral antibiotic treatment of right-sided staphylococcal endocarditis in injection drug users: prospective randomized comparison with parenteral therapy. Am J Med 1996;101:68-76.

49. Thuny F, Avierinos JF, Tribouilloy C, et al. Impact of cerebrovascular complications on mortality and neurologic outcome during infective endocarditis: a prospective multicentre study. Eur Heart J 2007;28:1155-61.

50. Hasbun R, Vikram HR, Barakat LA, et al. Complicated left-sided native valve endocarditis in adults: risk classification for mortality. JAMA 2003;289:1933-40.

51. Dickerman SA, Abrutyn E, Barsic B, et al. The relationship between the initiation of antimicrobial therapy and the incidence of stroke in infective endocarditis: an analysis from the ICE Prospective Cohort Study (ICE-PCS). Am Heart J 2007;154:1086-94.

52. Kang DH, Kim YJ, Kim SH, et al. Early surgery versus conventional treatment for infective endocarditis. N Engl J Med 2012;366:2466-73.

53. Prendergast BD, Tornos P. Surgery for infective endocarditis: who and when? Circulation 2010;121:1141-52.

54. Barsic B, Dickerman S, Krajinovic V, et al. Influence of the timing of cardiac surgery on the outcome of patients with infective endocarditis and stroke. Clin Infect Dis 2013;56:209-17.

55. Nappi F, Spadaccio C, Acar C. Use of allogeneic tissue to treat infective valvular disease: Has everything been said? J Thorac Cardiovasc Surg.2017;153:824-8.

56. Bando K. Does type of prosthesis affect long-term outcomes after aortic valve replacement for infective endocarditis? How should we properly answer this question? J Thorac Cardiovasc Surg 2017;153:829-30.

57. Nappi F, Spadaccio C. Simplest solutions are not always the cleverest: Can we stitch in an infected annulus? Should we rethink the current guidelines? J Thorac Cardiovasc Surg 2017;154:1899-900.

58. Bando K. Proposal for Building an Infrastructure for International Randomized Control Trials and a Comprehensive International Comprehensive Registry of Infective Endocarditis. J Thorac Cardiovasc Surg 2018;155:73-74.

59. Nappi F, Spadaccio C. Keep fumbling around in the dark when it comes to infective endocarditis, or produce new, reliable data to redesign the guidelines? J Thorac Cardiovasc Surg 2018;155:75-6.

60. Nappi F, Spadaccio C, Dreyfus J, et al. Mitral endocarditis: A new management framework J Thorac Cardiovasc Surg 2018;156:1486-1495.e4.

61. Nappi F, Nenna A, Petitti T, et al. Long-term outcome of cryopreserved allograft for aortic valve replacement. J 
Thorac Cardiovasc Surg 2018;156:1357-1365.e6.

62. Olivito S, Lalande S, Nappi F, et al. Structural deterioration of the cryopreserved mitral homograft valve. J Thorac Cardiovasc Surg 2012;144:313-20, 320.e1.

63. Chu VH, Park LP, Athan E, et al. Association between surgical indications, operative risk and clinical outcome in infective endocarditis: a prospective study from the International Collaboration on Endocarditis. Circulation 2015;131:131-40.

64. Ruttmann E, Willeit J, Ulmer H, et al. Neurological outcome of septic cardioembolic stroke after infective endocarditis. Stroke 2006;37:2094-9.

65. Musci M, Weng Y, Hübler M, et al. Homograft aortic root replacement in native or prosthetic active infective endocarditis: twenty-year single-center experience. J Thorac Cardiovasc Surg 2010;139:665-73.

66. Manne MB, Shrestha NK, Lytle BW, et al. Outcomes after surgical treatment of native and prosthetic valve infective endocarditis. Ann Thorac Surg 2012;93:489-93.

67. Kim JB, Ejiofor JI, Yammine M, et al. Are homografts

Cite this article as: Benedetto U, Avtaar Singh SS, Spadaccio C, Moon MR, Nappi F. A narrative review of the interpretation of guidelines for the treatment of infective endocarditis. Ann Transl Med 2020;8(23):1623. doi: 10.21037/atm-20-3739 superior to conventional prosthetic valves in the setting of infective endocarditis involving the aortic valve? J Thorac Cardiovasc Surg 2016;151:1239-46, 1248.e1-2.

68. San Román JA, Vilacosta I, López J, et al. Critical questions about left-sided infective endocarditis. J Am Coll Cardiol 2015;66:1068-76.

69. Gaca JG, Sheng S, Daneshmand MA, et al. Outcomes for endocarditis surgery in North America: a simplified risk scoring system. J Thorac Cardiovasc Surg 2011;141:98106.e1-2.

70. De Feo M, Cotrufo M, Carozza A, et al. The need for a specific risk prediction system in native valve infective endocarditis surgery. ScientificWorldJournal 2012;2012:307571.

71. Martínez-Sellés M, Muñoz P, Arnáiz A, et al. Valve surgery in active infective endocarditis: a simple score to predict in-hospital prognosis. Int J Cardiol 2014;175:133-7.

72. Kirklin JK. Challenging homografts as the holy grail for aortic valve endocarditis. J Thorac Cardiovasc Surg 2016;151:1230-1. 